\title{
Tire Defect Detection Based on Faster R-CNN
}

\author{
Zeju Wu ${ }^{1 *[0000-0001-7825-5802]}$, Cuijuan Jiao ${ }^{1[0000-0002-8735-6952]}$, Jianyuan $^{1[25]}$ Sun $^{2[0000-0001-8605-}$ \\ ${ }^{8825]}$ and Liang Chen ${ }^{10000-0002-6159-3888]}$ \\ ${ }^{1}$ The School of Information and Control Engineering, Qingdao University of Tech- \\ nology, Qingdao 266525, China. \\ ${ }^{2}$ National Centre for Computer Animation, Faculty of Media and Communication, Bourne- \\ mouth University, BH12 5BB Bournemouth, UK. \\ wuzeju_qut@163.com, sunj@bournemouth.ac.uk
}

\begin{abstract}
The tire defect detection method can help the rehabilitation robot to achieve autonomous positioning function and improve the accuracy of the robot system behavior. Defects such as foreign matter sidewall, foreign matter tread, and sidewall bubbles will appear in the process of tire production, which will directly or indirectly affect the service life of the tire. Therefore, a novel and efficient tire defect detection method was proposed based on Faster R-CNN. At preprocessing stage, the Laplace operator and the homomorphic filter were used to sharpen and enhance the data set, the gray values of the image target and the background were significantly different, which improved the detection accuracy. Moreover, data expansion was used to increase the number of images and improve the robustness of the algorithm. To promote the accuracy of the position detection and identification, the proposed method combined the convolution features of the third layer and the convolution features of the fifth layer in the ZF network (a kind of convolution neural network). Then, the improved ZF network was used to extract deep characteristics as inputs for Faster R-CNN. From the experiment, the proposed faster R-CNN defect detection method can accurately classify and locate the tire X-ray image defects, and the average test recognition rate is up to $95.4 \%$. Moreover, if there are additional types of defects that need to be detected, then a new detection model can be obtained by fine-tuning the network.
\end{abstract}

Keywords: rehabilitation robot, Faster R-CNN, improved ZF convolutional neural networks, recognition rate, tire defect detection.

\section{Introduction}

The tire defect detection method has a strong self-learning ability, which can help the robot achieve the function of auto-matic visual recognition [1-2]. It meets the requirements of rehabilitation robots for smart devices, makes medical services more accurate, and provides patients with more advanced and effective rehabilitation treatment processes [3]. A radial tire is widely recognized because it has the advantages of high speed, energy saving, durability, safety, comfort, and driving performance. The 
radial tire manufacturing process is complex and requires high precision. Quality problems often occur in the manufacturing process, which will directly or indirectly affect the service life of the tire, and even endanger personal safety [4-5]. Therefore, it is necessary to carry out the non-destructive test on each tire before leaving the factory, which can help persons quickly adjust the machinery production equipment in the tire production process. This test can save production material costs and improve tire quality.

At present, the tire defect detection methods generally employ an X-ray machine to obtain the images of the tire, and then workers observe the obtained images to identify whether the tires have the defects and classify these defects according to the shape and gray features of the images [6]. However, artificial visual inspection is an objective method. The detection results are easily affected by the level of workers' professionalism. It is easy to misjudge when the workload and intensity of the workers are large [7-8]. Therefore, in this paper, we propose a tire detection method based on Faster R-CNN. Using the convolutional neural network to obtain the feature point information of the image and analyze the upper semantics, and then replace the human eye to complete the task. It is applied to the research of rehabilitation robots, and it accomplishes a part of tasks that human eyes cannot judge competent in a special environment, and meets the needs of rehabilitation robots for high-precision positioning of smart devices. The tire detection system is used to realize the human eye guidance function of the rehabilitation robot, and the convolutional neural network and image processing technology are combined to complete the specific tasks of the rehabilitation robot in the given environment. The rapid development of artificial intelligence is of great significance to the development of rehabilitation robot automation.

In 2014, Ross Girshick et al. [9] designed the R-CNN target detection framework with a regional nomination strategy and CNN feature extraction algorithm, which made a great breakthrough in target classification and location tasks. To solve the problem of low efficiency and large training space of R-CNN, Girshick et al. [10] proposed the Fast R-CNN method. Firstly, Conv Character Map was obtained by carrying out the convolution operation of the image. At the same time, the region of interest (ROI) was obtained using the Selective Search method, and then the candidate region was mapped to the feature map of the CNN last layer. The image only needs to extract features once, which reduces the computational complexity. However, it still does not solve the problem of the slow computational speed of a selective search algorithm. The Faster R-CNN was proposed by Ren et al.[11], which used the region proposal networks (RPN) to select the proposal regions on the premise of absorbing the characteristics of Fast R-CNN. Moreover, most of the prediction is completed under the GPU, which greatly improves the detection speed and accuracy.

Organization of the paper as follows. Section 2 provides a review of the literatures regarding the development process of deep learning. In Section 3, we illustrate our algorithm elaborately. Section 4 provides the experimental results and Section 5 shows the conclusion of the paper. 


\section{Related Work}

In recent years, the problem of tire defect detection has attracted a lot of attention for domestic researchers. For example, Q Liu et al. [12] of Shandong University of Finance and Economics proposed a tire defect detection algorithm based on Radon transformation in 2015; Bin Zhang et al. [13] of Qingdao University Soft Control Enterprise proposed a tire X-ray image impurity detection technology based on image processing in 2016. The technology has been completed in four steps: histogram column equalization, Fourier transform and low-pass filtering, binarization and closed operation, and based on which the tire bead impurity defects were identified. However, these methods can only detect and identify the tire bead impurity defects. Xuehong Cui et al. [14] of Qingdao University of Science and Technology proposed a tire Xray image defect detection method based on inverse transformation of principal component residual information in 2017. The principal component analysis (PCA) is used to reconstruct the dominant texture of tire image, and then the defect can be found by subtracting the original image from the reconstructed dominant texture image (only using the small remaining eigenvalues and corresponding eigenvectors to restore the defect and noise). It can detect foreign matter sidewall, however, the extraction features are complex and require people to participate in the selection.

The above methods for tire defect detection are all based on traditional machine vision [15]. The features used for tire defect detection are artificially selected and designed [16], and the obtained features are jagged and poorly robust. Therefore, these methods are suitable for simple defect detection. The automatic recognition and localization of tire X-ray images with different defect areas, various shapes and complex background areas are powerless.

In 2006, Hinton et al. [17] proposed in-depth learning to solve this problem. Indepth learning can automatically learn the features of the target according to the training dataset, and abstract the high-level feature expression by integrating the transformation features of each layer from low to high, which makes classification and location easier. Since then, the emergence of excellent algorithms based on convolutional neural networks, and played a great role in target detection.

In this paper, in order to improve the recognition accuracy and the location accuracy, the tire defect location and recognition method is improved based on the Faster R$\mathrm{CNN}$. At preprocessing stage, the Laplace operator and the homomorphic filter are used to sharpen and enhance the data set due to the gray values of the image target and the background was significantly different. Moreover, we combine the convolution features of the third layer and the fifth layer of the ZF network as the input of the RPN layer. The convolution feature of the layer is combined as the input of the RPN layer; since the output of the third layer of the ZF network is 384 dimensions, and the output of the fifth layer is 256 dimensions, the convolution operation of is performed after the output of the third layer, so that its output is also 256 dimensions. The outputs of the layers are added.

Fig. 1 shows the specific process. Faster R-CNN network structure mainly consists of three parts: feature extraction layer, RPN (Regional Proposal Network) layer, Fast $\mathrm{R}-\mathrm{CNN}$ layer and so on. In the feature extraction layer, the ZF network is used to 
extract the feature map of the input image. The RPN layer is used to preliminarily extract regions of interest (ROI). Fast R-CNN layer is used to locate and identify tire defects.

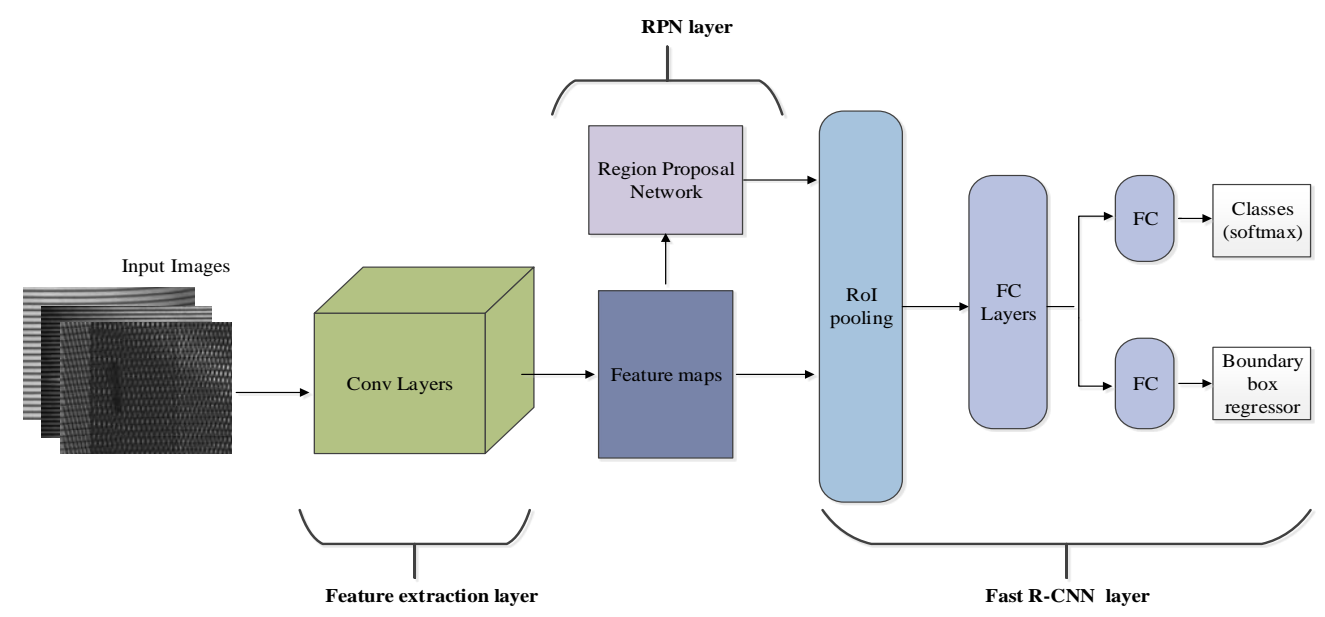

Fig. 1. Target detection framework based on Faster R-CNN

In this paper, the faster R-CNN and ZF network [10] (a kind of convolution neural network) is introduced to solve the task of the tire defect recognition and location, which provides a more concise and efficient method for industrial tire defect detection.

\section{Proposed Method}

\subsection{X-Ray Image Defect Detection Of Tire}

The X-ray images of tires mainly contain three types of defects: foreign matter sidewall, foreign matter tread and sidewall bubble, as indicated by the green arrow in Fig. 2. Fig. 2 shows the flowchart of the proposed method. The red arrow is rectangular boxes. The training set is pre-processed before network training: homomorphic filtering is used to enhance the training set. 


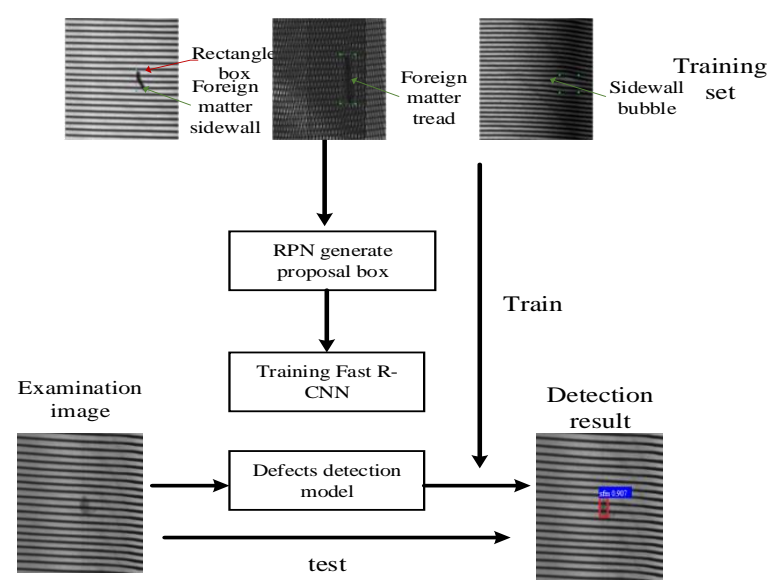

Fig. 2. Defect detection process based on Faster R-CNN

Using X-ray machine to make X-ray image of 360 degree tire irradiation, because the obtained X-ray image contains some noise. Target defects such as foreign matter sidewall and sidewall bubble, the gray difference between the target and background is very small in some areas. In this case, it is difficult for the human eye to find and recognize the target, which has negative for the labeling of the rectangular box and the training of the model, so it is necessary to do pre-processing to enhance the gray difference between these targets and background. This method is divided into two stages: training stage and testing stage.

Training stage: The input data of the training model was increased by the image geometric transformation method. And the rotation, the horizontal offset, the vertical offset, the scaling and horizontal flip were selected. The data augmentation method enables the convolutional neural network to learn more image invariant features and avoid overfitting. Defect detection model is obtained by determining the type of target to be detected (foreign matter sidewall, foreign matter tread, and sidewall bubble), selecting the network and training.

Testing stage: Testing the samples to be tested with the obtained model. The deep learning framework used in the experiment is MXNet, which is accelerated by GPU, and the feature extraction network is ZF. The image data used in this paper are provided by Soft Holding Company Limited.

Fig. 3 shows the labeled dataset sample. The tire defect detection dataset is constructed according to PASCAL VOC dataset format standard. The rectangular box is labeled on the image by Labelme. Fig. 3(a)-(d) show the foreign matter sidewall. Fig. 3(e)-(h) show the foreign matter tread. Fig. 3(i)-(l) show the sidewall bubble. An XML file is automatically generated for each image to record various information of the image, and the bounding box coordinate information. 

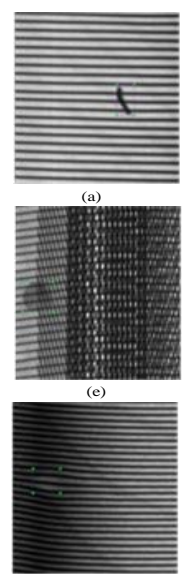

(i)
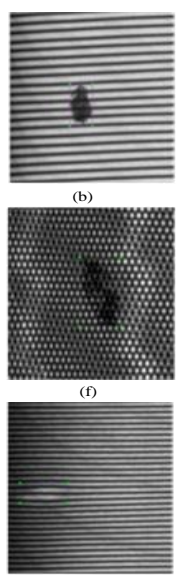
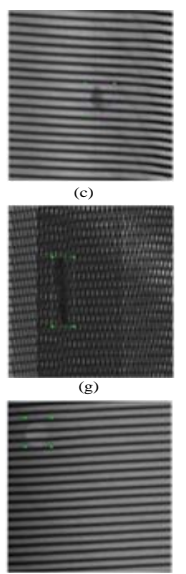
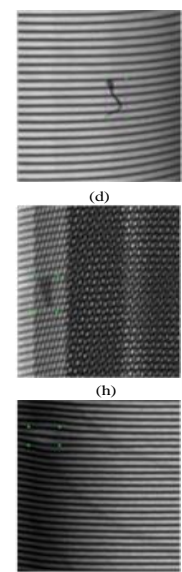

Fig. 3. Tire defect dataset sample

The training dataset contains 1022 pictures, including foreign matter sidewall (215), foreign matter tread (155), sidewall bubble (126) and normal tire X-ray images (including sidewall/ tread images) (526). The number of pictures in brackets represents the number of pictures. The proportion of each kind of defect sample is consistent with the frequency of occurrence of such defects. Defect image is the smallest rectangular image containing defects. Because of the uncertainty of defects in production, the length, width and size of defect image are different and distributed in $50 \times 50 \sim 200 \times 500$ pixel. In order to unify the size of defective images to meet the requirements of the algorithm, and to maximize the representation of image defects, while reducing computational complexity, In this paper, each image is segmented into the sidewall and tread regions, and the long sidewall and tread regions are divided into 8 segments and then the training dataset is composed of these images.

\section{Improved Convolutional Neural Network}

Convolutional neural network is specially designed for image recognition. It benefits from its network structure similar to biological neural network, which reduces the complexity of network model and the number of weights [18-21]. In this paper, ZF network is used to extract image features.

Fig. 4 shows the framework of the ZF network. The process of ZF network detection is:

- (1) The image of size is used as input, and the feature is extracted automatically by convolution layer;

- (2) Then the RPN is used to generate high quality proposal box, each image is about 300 proposal boxes;

- (3) The proposal boxes are mapped to the last convolution feature map of CNN;

- (4) The ROI pooling layer is used to fix the size of each proposal box; 
- (5) Classification layer and boundary regression layer is used to make specific classification judgment and accurate border regression for the proposal areas.

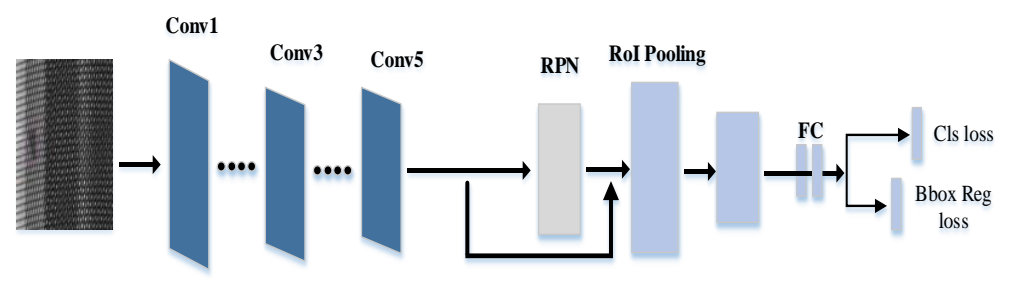

Fig. 4. Framework of the ZF network

Fig. 5 shows the framework of the improved ZF network. From the above detection process, we can see that only the last layer of convolution is used in the recognition of ZF network. Since the image information can be extracted into the abstract feature of the target after it passes through the multi-layer convolution layer, Although these abstract features are helpful for judging the specific categories of targets, after multi-layer convolution feature extraction, the details of the target will also be lost, which makes the extracted features less sensitive to the size and location of the target. Therefore, if only the last layer of convolutional feature information is used to locate and identify in the detection process, there will be a great error for the smaller defect area, because the smaller defect target (if the defect area is $25 \times 25$ pixel) has only one or several convolution features after passing through five layers of convolution layer. Such little information not only has a negative impact on location precision, but also influences the recognition of the target.

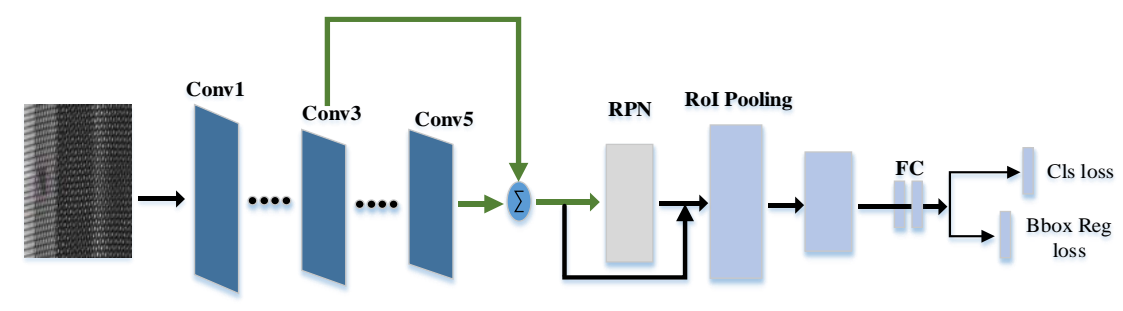

Fig. 5. Framework of the improved ZF network

According to the above situation, inspired by the Fully Convolutional Networks (FCN) on the success of the Semantic Segmentation task proposed by Jonathan Long et al. [22], to improve the recognition accuracy and location accuracy, the tire defect location and recognition method is improved based on Faster R-CNN. Based on the Faster R-CNN network, this paper combines the convolution features of the third layer and the fifth layer of ZF network as the input of RPN layer. The convolution feature of the layer is combined as the input of RPN layer; since the output of the 
third layer of ZF network is 384 dimensions, and the output of the fifth layer is 256 dimensions, the convolution operation of is done after the output of the third layer, so that the output of the third layer is 256 dimensions, and then the output of the fifth layer is added. Because the shallower convolution layer can extract local features in the feature extraction process of the target detection network, the deeper convolution layer can extract more abstract features, if the features of multiple different layers can be combined, it will help target detection [23].

The purpose of improving ZF network in this paper is to avoid gradient explosion, improve learning rate, and extract double depth features of defect samples to improve the classification performance of the network.

\section{Region Proposal Network}

Fig. 6 shows the framework of the Region Proposal Network (RPN). The RPN is a full-convolution network. The RPN network can quickly generate anchor boxes of different sizes, and determine the probability of the target or background of the image in the frame for preliminary extraction of Region of Interest (ROI), which solves the speed problem of the Selective Search (SS) [24], and greatly improves the target detection speed. The specific process of constructing the RPN is to use a small sliding window ( Convolution Kernel) to scan the feature map of the final convolution. After sliding convolution, a d-D vector is mapped. Finally, the d-D vector is sent to two fully connected layers, the regression layer and the classification layer. In each sliding window, region proposal boxes are predicted at the same time, so the regression layer has outputs to encode the coordinates of region proposal boxes, and the classification layer outputs scores to estimate the probability that each region proposal box is a target/non-target.

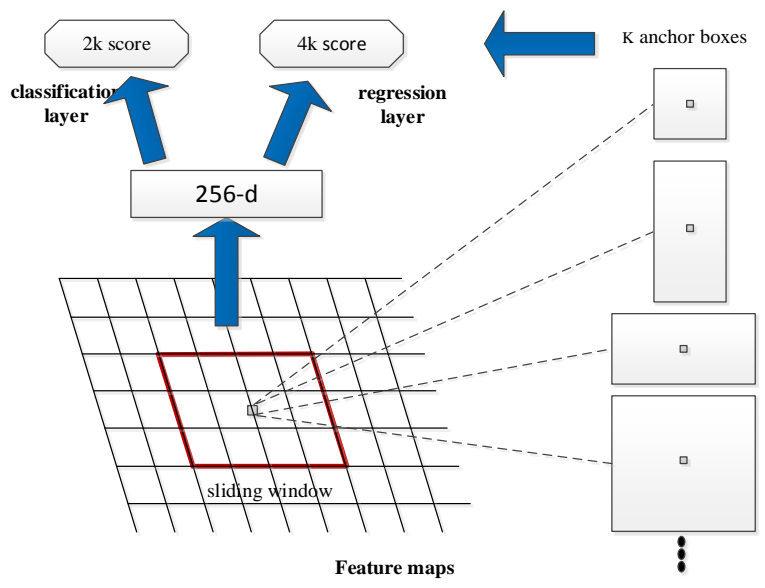

Fig. 6. Framework of the Region Proposal Network

The RPN network uses a Bounding Box Regression (BBox) to generate an ROI, which loss function is defined as (1). 


$$
L_{r e g}\left(t_{i}, t_{i}^{*}\right)=\sum_{i=x, y, w, h} \operatorname{Smooth}_{L_{1}}\left(t_{i}-t_{i}^{*}\right)
$$

Where, $\mathrm{x}, \mathrm{y}, \mathrm{w}, \mathrm{h}$ represents the central coordinates of ROI and their width and height, $t_{i}$ represents four parametric coordinates of the predicted bounding box, $t_{i}{ }^{*}$ represents the coordinate vector of the Ground Truth (GT) bounding box corresponding to the positive anchor. For robust loss function in 错误!未找到引用源。 is shown in (2) and (3).

When $|x|<1$ :

$$
\operatorname{Smooth}_{L_{1}}(x)=0.5 x^{2}
$$

$|x| \geq 1:$

$$
\operatorname{Smooth}_{L_{1}}(x)=|x|-0.5
$$

The total loss function of the RPN network is defined as (4).

$$
L\left(\left\{P_{i}\right\}\left\{P_{i}^{*}\right\}\right)=\frac{1}{N_{c l s}} \sum_{i} L_{c l s}\left(P_{i}, P_{i}^{*}\right)+\lambda \frac{1}{N_{r e g}} P_{i}^{*} \sum_{i} L_{r e g}\left(t_{i}, t_{i}^{*}\right)
$$

Where, $i$ represents the window index value generated by RPN in a single image sample feature graph, $P_{i}$ represents probability indicating that the window is a target, $P_{i}{ }^{*}$ represents the predictive probability of the GT, $N_{c l s}$ is the normalized value of the classification term, i.e. the batch number $256 ; N_{\text {reg }}$ is normalized value of regression term, $\lambda$ is weight value used to balance the two kinds of losses. In this paper, we set $\lambda=1$ to make the two weights approximately equal.

\section{Fast R-CNN Network}

In practical calculation, 1000 ROIs are provided by RPN network, and 300 of them are randomly selected for Fast RCNN network training. The positive and negative samples are judged by Intersection over Union (IOU) [25]. In order to ensure the quality of positive samples, when IOU $>0.6$, the ROI is determined to be positive sample; when IOU $<0.2$, it is negative sample. Fast R-CNN uses pooling to process ROI of different sizes to ensure that input vector dimensions are the same when entering the full connection layer. ROI pooling input is divided into two parts, RPN network output proposals and CNN network output image feature map. Proposals correspond to the $M \times N$ scale, so firstly, it is mapped back to the $(M / 16) \times(N / 16)$ scale by using the spatial scale number of $1 / 16$. Then the horizontal and vertical directions of each proposal are divided into seven equal parts, and each proposal is processed by 
Max pooling. After processing, proposals of different sizes are all $7 \times 7$ in size, which achieves fixed length output.

The proposal feature maps were obtained using the ROI pooling, and the full connection layer and softmax calculate the category of each proposal (foreign matter sidewall and foreign matter tread, sidewall bubble), and outputs the category probability vector. At the same time, the positional offset was obtained using BBox, which is used to return a more accurate target detection frame.

\section{4 . Experimental Results and Analysis}

\subsection{Analysis of Preprocessed Results}

Fig. 7(a) shows the original image, and Fig. 7(b) shows its corresponding histogram. It can be seen from the original image that the defect contains the foreign matter tread. Some defects are very close to the gray value of the background, and the gray distribution is relatively scattered. Fig. 7(c) shows the image after preprocessing, and Fig. 7(d) shows the histogram after preprocessing. After processing, the gray value of the target and background of the image has obvious difference, and the gray distribution is more concentrated.

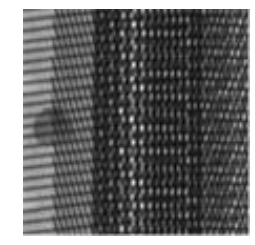

(a)

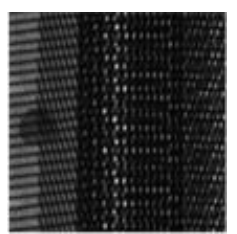

(c)

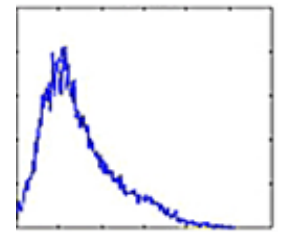

(b)

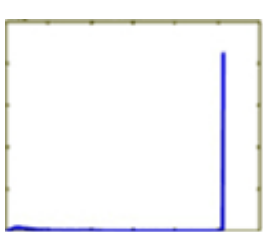

(d)

Fig. 7. The preprocessed results: (a) The original image, (b) The corresponding histogram, (c) The preprocessed image, and (d) the histogram of the preprocessed image.

\subsection{Defect Detection Results}

Fig. 8 shows the results of image detection using an improved Faster R-CNN network model.

Fig. 8(a) shows the detection results of the foreign matter sidewall, Fig. 8(b) shows the detection results of the foreign matter tread, and Fig. 8(c) shows the detection results of the sidewall bubble. It can be seen that for three kinds of defects of different 
sizes, the test result shows that the detection quality is high. Fig. 8(d) shows the result of false detection. The yellow area in the picture is the real location of the bubble defect, and the normal area in the picture is mistake for as the bubble defect (such as the red box). This is mainly due to the fact that the weak edges of bubble defects in Xray images can hardly be clearly displayed, while the detection accuracy of bubble defects with strong edges like Fig. 8(c) is higher.

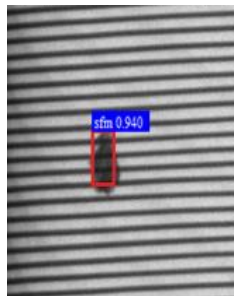

(a)

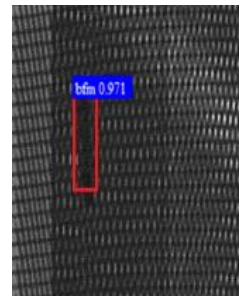

(b)

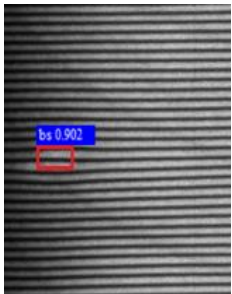

(c)

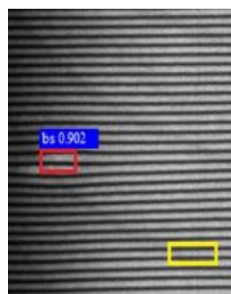

(d)

Fig. 8. The detection results (a) The detection results of the foreign matter sidewall, (b) The detection results of the foreign matter tread detection, (c) The detection results of the sidewall bubble, and (d) The result of false detection.

The total number of pictures to be detected is 184 . Table 1 shows the number statistics of test results, include foreign matter sidewall, foreign matter tread and sidewall bubble defects. And Table 2 shows the ratio statistics of test results.

Table 1. The number statistics of test results

\begin{tabular}{lccc}
\hline \multicolumn{1}{c}{ Defects } & Correct & Missed & False \\
\hline Foreign matter sidewall & 36 & 1 & 0 \\
Foreign matter tread & 55 & 2 & 0 \\
Sidewall bubble & 33 & 3 & 2 \\
Total & 124 & 6 & 2 \\
\hline
\end{tabular}

Table 2. The ratio statistics of test results

\begin{tabular}{lccc}
\hline \multicolumn{1}{c}{ Defects } & Correct rate & Missed rate & False rate \\
\hline Foreign matter sidewall & 0.973 & 0.027 & 0 \\
Foreign matter tread & 0.965 & 0.035 & 0 \\
Sidewall bubble & 0.917 & 0.083 & 0.056 \\
Total & 0.954 & 0.046 & 0.015 \\
\hline
\end{tabular}

From Table 1 and Table 2, it can be seen that the method has good detection results for all three kinds of defects. It has a higher detection rate for other defects except for some bubble defects. 


\subsection{Comparison of Test Results}

In the experiment, mAP(mean average precision) [25] is used as the accuracy evaluation index of tire defect detection performance. The higher the value is, the higher the detection accuracy of the algorithm is.

Table 3 shows the comparison results of the different method. The detection accuracy of the original algorithm is $85.17 \%$, and the speed is $17 \mathrm{fps}$. Add the preprocessing method to the original algorithm, the detection accuracy is $93.82 \%$ and the speed is $19 \mathrm{fps}$. The method in this paper, the model detection accuracy is $95.37 \%$ and the speed is $28 \mathrm{fps}$. Although the accuracy of the method in this paper is slightly improved from $93.82 \%$, the detection speed is greatly improved, and the detection effect is better.

Table 3. Comparison of test results of different methods

\begin{tabular}{lcc}
\hline Method & mAP/\% & Speed/fps \\
\hline Original algorithm & 85.17 & 17 \\
Original algorithm +Preprocessed & 93.82 & 19 \\
Method in this paper & 95.37 & 28 \\
\hline
\end{tabular}

The training results of in [10] algorithm and the results of direct detection after preprocessed are compared with the accuracy of the detection results of this method. Table 3 and Iv show the specific comparison results.

Fig. 9 shows the detection results in [10] algorithm, Fig. 9(a) shows the detection results of the foreign matter sidewall, Fig. 9(b) shows the detection results of the foreign matter tread, and Fig. 9(c) shows the detection results of the sidewall bubble. The algorithm in [10] is poor for detection of foreign matter tread and sidewall bubble.

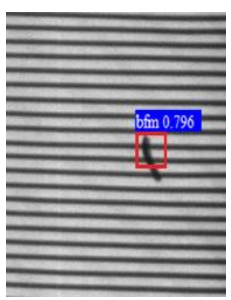

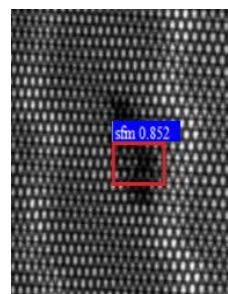

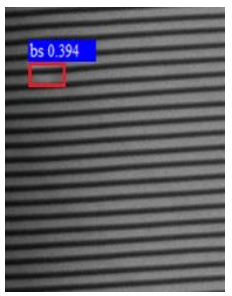

Fig. 9. The detection results in [10] algorithm (a) The detection results of the foreign matter sidewall, (b) The detection results of the foreign matter tread, (c) The detection results of the sidewall bubble. 
Fig. 10 shows the detection results of preprocessing tests, Fig. 10(a) shows the detection results of the foreign matter sidewall, Fig. 10(b) shows the detection results of the foreign matter tread, and Fig. 10(c) shows the detection results of the sidewall bubble. The pretreatment algorithm has greatly improved the sidewall bubble.

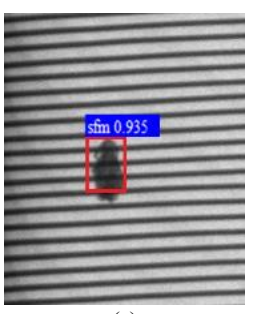

(a)

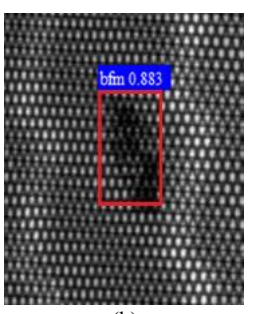

(b)

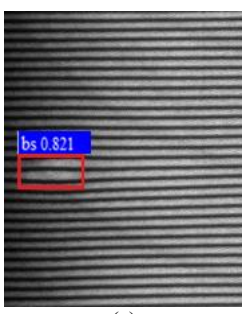

(c)

Fig. 10. The detection results of preprocessed tests (a) The results of the foreign matter sidewall detection, (b) The results of the foreign matter tread detection, and(c) The results of the sidewall bubble detection.

Fig. 11 shows the comparison of the detection accuracy. The histogram of the detection accuracy obtained from the statistical results in Table 4 and 5. From Fig. 11, it can be seen that the three methods have high detection accuracy for foreign matter sidewall, but algorithm used in [10] is poor for detection of foreign matter tread and sidewall bubble. Because of the preprocessing of the training dataset and the improvement of the ZF network, the accuracy of detecting foreign matter tread and sidewall bubble has been greatly improved.

Table 4. The number statistics of test results in [10]

\begin{tabular}{lccc}
\hline \multicolumn{1}{c}{ Defects } & Correct & Missed & False \\
\hline Foreign matter sidewall & 29 & 5 & 3 \\
Foreign matter tread & 11 & 3 & 1 \\
Sidewall bubble & 10 & 16 & 4 \\
Total & 50 & 24 & 8 \\
\hline
\end{tabular}

Table 5. The number statistics of preprocessed test results

\begin{tabular}{lccc}
\hline \multicolumn{1}{c}{ Defects } & Correct & Missed & False \\
\hline Foreign matter sidewall & 62 & 5 & 1 \\
Foreign matter tread & 50 & 6 & 3 \\
Sidewall bubble & 32 & 7 & 2 \\
Total & 144 & 18 & 6 \\
\hline
\end{tabular}




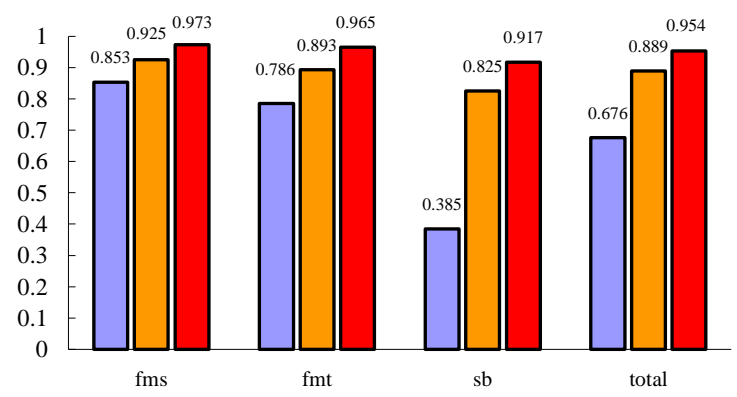

Fig. 11. Comparison of the detection accuracy of different methods

In Fig. 11, the blue part represents the accuracy of the original algorithm, the yellow part represents the accuracy of the method of add preprocessing to the original algorithm, and the red part represents the accuracy of the method in this paper.

\section{Conclusions}

This paper proposes a novel tire defect detection method based on Faster R-CNN, which is used to identify the type of tire defects and mark the location of the defects. In the pre-processing stage, the homomorphic filtering method is used to sharpen and enhance the training dataset, which avoids the generation of a large number of redundant windows and improves the detection speed and accuracy. By improving the framework of $\mathrm{ZF}$ network and referring to the structure of semantic segmentation network FCN, combining the third-level convolution feature with the fifth-level convolution feature, smaller defects can be detected. The target improves the accuracy of location detection and recognition in Faster R-CNN network. Compared with traditional methods, the detection process has been greatly improved in the degree of automation, and has strong robustness, which is an effective and feasible method. In the future, this method can be applied to the intelligent manufacturing of rehabilitation robots to improve the core competitiveness of enterprises.

At present, the method studied in this paper can accurately locate and identify defects, but the results of the bubble location are slightly poor. In the future, we will combine a variety of direct and non-direct detection methods to conduct in-depth research on bubble defects.

\section{Acknowledgment}

This work was supported by the Key Research and Development Program of Shandong Province (NO.2018GGX101040), the Applied Basic Research Programs of Qingdao (NO. 18-2-2-62-jch), the National Natural Science Foundation of China 
(NSFC) (No.61501278), the Natural Science Foundation of Shandong Province (No.ZR2015FQ013). There are no conflicts of interest.

\section{References}

1. Hou Z G, Zhao X G, Cheng L, Wang Q N, Wang W Q (2016) Recent advances in rehabilitation robots and intelligent assistance systems. Journal of Automation, vol. 42, nn.12, pp. 1765-1779. doi:10.16383/j.aas.2016.y000006.

2. Gu T, Li C J, Zhan Q (2017) Advances in application of rehabilitation robots for upper limb dysfunction in patients with stroke. Journal of neurology and neurorehabilitation, vol. 13, nn.1, pp. 44-50.

3. Zheng X Z (2018) Take the intelligent rehabilitation training robot as an example to analyze the influence of artificial intelligence on rehabilitation sports technology. The road to health (2), pp. 9-9.

4. Wu G H, Xiong H J (2016) Current situation and research progress of radiographic testing technology in China. Instrumentation Journal, vol. 37, nn.8, pp. 1683-1695. doi: 10.3969/j.issn.0254-3087.2016.08.001

5. Wu Z J, Lin J J, and Liu W J (2019) Joint inspection in X-ray \#0 belt tire based on periodic texture. Multimedia Tools \& Applications, vol. 78, nn. 7, pp. 9299-9310.

6. Yan W X (2016) Research on deep learning and its application on the casting defects automatic detection. Guangzhou: South China University of Technology. doi:CNKI:CDMD:2.1016.770553

7. Zhang Y (2014) Research on key problems of tire defect nondestructive testing based on computer vision. Qingdao University of Science and Technology.

8. Zhang Y, Lefebvre D, and Li Q (2017) Automatic Detection of Defects in Tire Radiographic Images. IEEE transactions on automation science \& engineering, vol. 14, nn. 3, pp. 1378-1386. doi: 10.1109/TASE.2015.2469594

9. Girshick R, Donahue J, Darrell T, et al. (2014) Rich feature hierarchies for accurate object detection and semantic segmentation, Proceedings of 2014 IEEE Conference on Computer Vision and Pattern Recognition. Columbus, OH, USA: IEEE, pp. 580-587.

10. Girshick R (2015) Fast R-CNN[C].In: 2015 IEEE International Conference on Computer Vision. Santiago, pp. 1440-1448.

11. Ren S, He K, Girshick R, et al. (2017) Faster R-CNN: towards real-time object detection with region proposal networks. IEEE Transactions on Pattern Analysis and Machine Intelligence, vol. 39, nn. 6, pp. 1137-1146. doi: 10.1109/TPAMI.2016.2577031

12. Liu Q, Wang G, Guo Q, Liu Y, Zhang C (2015) Tire Defect Detection Based on Radon Transform. Computational Information Systems, vol. 11, nn. 21, pp. 7841-7848.

13. Zhang B, Lin S, Gao S (2016) Impurity detection technology of tire X-ray image based on image processing. Rubber technology and equipment, vol. 42, nn. 9, pp. 50-54.

14. Cui X H, Liu Y, Wang C X (2016) Defect automatic detection for tire X-ray images using inverse transformation of principal component residual. In: Artificial Intelligence \& Pattern Recognition. pp. 1-8. doi: 10.1109/ICAIPR.2016.7585205

15. Alaknanda, Anand R S, Kumar P (2009) Flaw detection in radiographic weldment images using morphological watershed segmentation technique. NDT \& E INTERNATIONAL, vol. 42, nn. 1, pp. 2-8. doi: 10.1016/j.ndteint.2008.06.005

16. Bai F, Zhang M L, Zhang X J, et al. (2016) A survey of local binary feature description algorithms. Electronic measurement and instrument, vol. 30, nn. 2, pp. 165-178. doi: 10.13382/j.jemi.2016.02.001 
17. Hinton G E (2006) Reducing the dimensionality of data with neural networks. Science, vol. 313, nn. 5786, pp. 504-507. doi: 10.1126 /science. 1127647

18. Zeiler M D, and Fergus R (2013) Visualizing and Understanding Convolutional Networks. In: Computer Vision Springer, pp. 818-833. doi: 10.1007/978-3-319-10590-1_53

19. Abou-Ali M G, Khamis M (2003) TIREDDX: an integrated intelligent defects diagnostic system for tire production and service. Expert Systems with Applications, vol. 24, nn. 3, pp. 247-259. doi: 10.1016/s0957-4174(02)00153-7

20. Ren L L, An D F, Shen Y D (2004) Causes for defects of carcass ply cord in TBR tire and their counter measures. Tire industry, vol. 24, nn. 9, pp. 559-561.

21. Li J N, Zhang B H (2018) Face recognition by feature matching fusion combined with improved convolutional neural network. Laser \& Optoelectronics Progress, vol. 55, nn. 10, pp. 246-253.

22. Long J, Shelhamer E, Darrell T (2014) Fully convolutional networks for semantic segmentation. IEEE Transactions on Pattern Analysis \& Machine Intelligence, vol. 39, nn. 4, pp. 640-651. doi: 10.1109/TPAMI.2016.2572683

23. Yang X M, Wu W, Qing L B, et al. (2009) Image feature extraction and matching technology. Guangxue Jingmi Gongcheng Optics and Precision Engineering, vol. 17, nn. 9, pp. 2276-2282. doi: 10.1360/972009-1549

24. Xu L M, Lu J D (2015) Bayberry image segmentation based on homomorphic filtering and K-means clustering algorithm. Transactions of the Chinese Society of Agricultural Engineering, vol. 31, nn. 14, pp. 202-208. doi: 10.11975/j.issn.1002-6819.2015.14.028

25. Feng X Y, Mei W, Hu D S (2018) Aerial target detection based on improved Faster RCNN. Acta Optica Sinica, 38(6): 0615004. 